\title{
David Viñas: antropofagia, tango y fachada
}

\section{(1) Carlos García}

Investigador independiente. Hamburgo, Alemania

Según informé en otra oportunidad (García agosto 2017), preparo la edición comentada de la correspondencia que Viñas mantuvo con el profesor alemán Dieter Reichardt entre 1977 y 1990. El corpus está conformado por 90 cartas y por varios textos de Viñas, tanto manuscritos como mecanografiados, así como recortes de publicaciones hemerográficas. La mayoría de las cartas son del argentino; del alemán solo se conservan unos pocos borradores. Todas las misivas son inéditas hasta ahora.

De ese trabajo en curso, deseo desgajar algunos pasajes, y presentar un texto de Viñas desconocido en Argentina.

En 1990, Viñas y Reichardt participaron en un coloquio sobre "La literatura de las grandes ciudades", que tuvo lugar en Berlín entre el 14 y el 16 de junio. Entre ambos tuvieron a su cargo la "Sección Buenos Aires": el primero con "Buenos Aires: fundación, antropofagia y continuidad"; el segundo con "Literatura de Buenos Aires". Ambos textos figuran en las Actas del congreso, en una edición de corta tirada aparecida décadas atrás en Alemania (Daus ed., 1992: 77-88).

En ese libro se reproduce en página 76 la imagen de una versión anterior, muy sumaria, provisoria y castigada, del texto de Viñas: un casi ilegible bosquejo de apenas una página firmado en "Berlín, 12-VI-90" (es decir, dos días antes de que comenzara el Coloquio). Debe haber servido de ayuda-memoria. Aparecen ya en ese breve apunte varios de los términos y conceptos que Viñas utilizará en la versión final, puesta por escrito, al parecer, tras el coloquio, quizás en base a una desgrabación (tal fue el caso con la ponencia de Reichardt).

Se conserva también el enrevesado manuscrito que sirviera de base a la publicación, y que obra ahora en mi poder (me fue obsequiado por Reichardt el 8-XII-2007). Lleva firma de Viñas y la fecha "19-VI-90", y el agregado "por borrador". Permite completar lagunas y corregir graves errores de lectura de quienes tuvieron a su cargo el establecimiento del texto para su impresión en el volumen arriba mencionado.

Reproduzco a continuación el texto de Viñas. Dejo constancia en nota al pie de las variantes importantes que ostenta el manuscrito en relación con el texto impreso. Este no considera la repartición en párrafos del hológrafo, aquí restituida. Todas las notas al pie son mías. 


\section{David Viñas \\ Buenos Aires: Fundación, antropofagia y continuidad}

\author{
[Großstadtliteratur, 1992: 77-82]
}

Si tuviese que trazar, hipotéticamente, una línea longitudinal desde el año de fundación de Buenos Aires en 1536 hasta esta década final del siglo XX, a los efectos de insinuar un componente central, aludiría a la antropofagia que comenta Luis de Miranda en aquel momento inaugural. ${ }^{1}$

1. Alusión al "Romance Elegíaco" de fray Luis de Miranda (hacia 1540): “Allegó la cosa á tanto/ Que, como en Jerusalén,/ La carne de hombre también/ La comieron./ Las cosas que allí se vieron/ No se han visto en escritura,/ Comer la propia asadura/ De su hermano". Véase la edición de Bernardo Canal Feijóo (1979), quizás la edición que conocía DV. Una más moderna y científica: El Romance de Luis de Miranda. Ed. de Silvia Tieffemberg (2012) (aquí, versos 81-84). Hay una posterior, que no he visto, a cargo de la misma persona: Romance (2014). La editora, académica argentina, tiene en su haber otras publicaciones relacionadas con la época colonial, entre ellas: Argentina. Historia del Descubrimiento y Conquista del Río de la Plata de Ruy Díaz de Guzmán (2012).

2. Una tesis similar, basada en Viñas, Mujica Láinez, Cortázar y otros, sostiene Jorge Monteleone en "La vaca o la res argentina", ensayo incluido en Comidas bastardas: Gastronomía, tradición e identidad en América Latina (2013).

3. "el lansquenete alemán" en el manuscrito. En Indios, ejércitos y frontera (1982: 89), Viñas cita a Schmidl y lo caracteriza como "cronista del siglo XVl". Extraña que no aproveche en ese libro a Schmidl como testigo en relación con la "guerra de la carne" de que allí se ocupa. 4. Sobre esta fecha, que es la de la primera fundación de Buenos Aires, es el informe de Schmidl. Hay una excelente nueva edición del libro (aparecido por primera vez en 1567), a cargo de Loreley El Jaber (2016): Ulrico (Utz) Schmidl: Derrotero y viaje a España y las Indias. El volumen se ve enriquecido con varios apéndices de la pluma de El Jaber y por numerosas ilustraciones de época. Véase igualmente la edición crítica del manuscrito alemán en Franz Obermeier: Ulrich Schmidel / Ulrico Schmidl: Reise in die La PlataGegend (1534-1554). Kritische Ausgabe (2008) (contiene también la traducción de Wernicke, en la versión de 1948, y numerosos apéndices).
Sobre todo que Miranda no sólo es un clérigo que funciona como Capellán de Pedro de Mendoza, primer adelantado y fundador, sino que es el comentarista, en verso, de las carencias y padecimientos de los soldados españoles como consecuencia del incendio de la primitiva fundación por los indios humillados y exigidos en calidad de sirvientes.

Servidumbre, rebeldía e incendio por el lado de los indios, entonces, que si desembocan en hambre culminan en antropofagia entre los mismos españoles. Centro de gravedad, por consiguiente, de un drama que, en mi criterio, se va repitiendo desde el siglo XVI hasta el 1990 de Buenos Aires. Y que define mediante sus diversas inflexiones (sobre todo en la franja de la literatura), lo que podría considerarse la divisa mayor de mi ciudad. ${ }^{2}$

Acortando distancias: aquella dramaturgia fundadora que, además de Miranda fue comentada por el alemán ${ }^{3}$ Ulrico Schmidl, hoy se verifica no solo en la despiadada represión producida entre 1976 y el 83 bajo la dictadura militar, sino en esa especie de obscena prolongación que se instaura entre una "cultura de fachada" y una tragedia disimulada en los sótanos del Shopping Center de Viamonte y Florida: triunfalismo y sordidez podrían ser, por consiguiente, los emblemas de ese circuito diacrónico abierto en $1536 .{ }^{4}$

Se trata, como vamos viendo, de una suerte de coreografía urbana que oscila entre lo institucional y lo clandestino, lo oficial y lo solapado, lo espectacular y lo silenciado. Dicotomía fundamental que va adoptando diversos matices e inflexiones en el plano simbólico.

Tanto es así que si la ciudad de Buenos Aires, incluso después de su segunda fundación en 1580, parece adormecerse en eso que suele llamarse "siesta colonial" que se prolonga a lo largo del siglo XVII y gran parte del XVIII, en el revés de trama de esa somnolencia aldeana, corresponde ir viendo "la multiplicación de los ganados", cuyos síntomas apenas si superan los rumores de la naturaleza.

Buenos Aires entre el 1600 y el 1750 no tiene el brillo cortesano que ostentan los viejos virreinatos Habsburgo: en México y en Lima, se sabe, el discurso colonial va discurriendo entre las cortesanías, el barroco, el juego escolástico y los predominios mineros. Buenos Aires no tiene barroco, sutilezas ni minería; apenas si las vacas mugen, proliferantes entre los suburbios y los cardales.

Recién cuando esos vacunos se han transformado en millones y sus cueros en la única industria, Buenos Aires se va despegando de la modorra hasta convertirse en virreinato. $\mathrm{Y}$ en esta mutación desde ya que incide además de la acumulación del cuero, la inquietante expansión británica del siglo XVIII, así como la aburguesada racionalidad de los Borbones españoles.

Racionalidad borbónica que va definiendo los rasgos primordiales de un Buenos Aires como virreinato borbónico. Cuya emergencia cultural más notoria, lograda a través -lógicamente- del virrey más iluminado, será el "Teatro de la Ranchería". 
Fundación escénica que vendrá a corroborar-con su edificio y su final- ese itinerario dramático con que pretendo enlazar a 1536 y a 1990. Entendámonos: el primer teatro de Buenos Aires fue incendiado hacia el 1790 por quienes se oponían a la iluminación de la ciudad; 5 en $1982^{6}$ se produce algo similar: el teatro de la calle Rauch (entre Callao y Corrientes) también fue incendiado por quienes se oponían a cualquier "esclarecimiento" de Buenos Aires. ${ }^{7}$

Si se focalizara ${ }^{8}$ el emergente literario de ese momento, correspondería hablar de Lavardén, quien, sobre el 1800, condensa la mayor producción simbólica en la poesía y el teatro: porque si su Oda al Paraná implica el primer poema vinculado al eje de la ciudad, Siripo es el drama que mejor sintetiza en esa circunstancia a los conflictos decisivos de Buenos Aires. Con un rasgo fundamental: la oda aparece frontalmente como una exhortación a que el gran río salga de su estancamiento y apunte hacia la movilidad. Inercia y movimiento son los extremos que tensan esa escenografía neoclásica que parece condenada a la decoración.

Pero lo que vibra por debajo de esa monumentalidad es la urgencia por que el río se abra. ¿A qué? En el plano de lo concreto, al "comercio libre", operación inaugural, y quizás insidiosa, que por esas fechas presuponía, ante todo, el privilegio acordado al "negocio de mayor movilidad", es decir, al comercio de esclavos. Benemérita actividad a la que estaba íntimamente vinculado Lavardén por sus compromisos comerciales con Tomás Romero, el mayor negrero rioplatense de fines del siglo XVIII y comienzos del XIX. De donde se puede inferir, quizás, ${ }^{9}$ al recorrer los ejemplares polvorientos del primer ${ }^{10}$ periódico del Buenos Aires de entonces, el Telégrafo Mercantil, Rural, Político e Historiográfico del Río de la Plata entre 1801 y 1802.

La poesía inicial porteña, como se ve, parece balancearse entre una fachada ornamental y un trasfondo abyecto. $Y$ así como en los alrededores del 180o, Lavardén sintetiza una serie de signos de la cual es su emergente y condensador, dibujando un ademán que parece preanunciar esa frase de Borges que insinúa la definición del tango esencial de cada "generación" como una necesidad de lectura, irónica, de la íntegra colección de El alma que canta. ${ }^{11}$

Correlativamente, el "tango esencial", en corte sincrónico, de la producción simbólica adscripta al Buenos Aires revolucionario de 1810, se constituye a su vez, sobre dos textos muy discutidos de Mariano Moreno: la Representación de los hacendados y el Plan de operaciones. ${ }^{12} \mathrm{Y}$ en este caso los emblemas se articulan entre la máscara y el don: porque si el encubrimiento se nos aparece como residuo del vasallaje colonial respecto de Fernando VII (en virtud de necesidades tácticas de la coyuntura histórica), el reconocimiento entretejido con el "don" "13 que se les otorga a los esclavos ${ }^{14}$ que entraban al servicio de la revolución, presuponía una ampliación de la base social subversiva y, al mismo tiempo, el [tránsito desde la \{dos palabras ilegibles\} hacia el develamiento y la reci]procidad. ${ }^{15}$ Por más de una razón, esta renovación del perfil porteño lo fue situando a Moreno, cada vez más hasta su exclusión y su exilio, en medio de la secuencia de la izquierda revolucionaria de 1810 que a nivel latinoamericano, implica también a "exiliados" como Hidalgo, Morelos, Sucre, Monteagudo, Artigas (con la secuela de símbolos que aluden, desde ya, a las respectivas ciudades).

Podría enunciar así: "el tango esencial" que alude al Buenos Aires de 1850 es, ineludiblemente, el Facundo con el interjuego, lúcido en sus mejores momentos, entre la constelación urbana y la rural. Inscripto, a su vez, en el período sarmientino que va del 1845 al 1852 y que se articula entre Recuerdos de provincia, los Viajes, Campaña en el ejército grande, Argirópolis por supuesto, hasta recalar en la polémica con Alberdi. Trayecto que, en su núcleo, se juega entre el ahorro y el despilfarro, la salud y la
5. Según la versión recibida, un cohete tirado desde una iglesia quemó el techo de paja del teatro. 6. “1982" en el manuscrito; en el texto impreso, por error de lectura, "1962". 7. El teatro "El Picadero", ubicado en el Pasaje Discépolo (antes Rauch) 1857, que había sido inaugurado por Antonio Mónaco en 1980. DV data mal el hecho: el teatro fue incendiado no en 1982, sino el 6-VIII-1981, debido a su programa de corte libertario. Fue reabierto en el 2012. (Curiosamente, la palabra "Rauch" significa "humo" en alemán.) Dice Piglia en Los diarios de Emilio Renzi, III, 2017, 145: “Anoche atentado contra El Picadero, donde se desarrollaba Teatro Abierto. Nos juntamos en la cortada, somos un grupo indignado y confuso". 8. "focalizara" en el manuscrito; "focalizaría” en el texto impreso (errata). 9. “, quizás,” no figu-

ra en el manuscrito. 10. "1er" en el manuscrito; "perdido" en el texto impreso. De un modo u otro, la frase es algo malsonante, como si hubiera algún otro error de escritura o transmisión. 11. Revista sobre tango fundada por Vicente Bucchieri; apareció entre 1916 y 1961. Véase Ariel Lomba (1998). Lo que Borges había dicho al respecto es esto: "es verosímil que hacia 1990 surja la sospecha o la certidumbre de que la verdadera poesía de nuestro tiempo no está en La urna de Banchs o en Luz de provincia de Mastronardi, sino en las piezas imperfectas que se atesoran en El alma que canta. Esta suposición es melancólica. Una culpable negligencia me ha vedado la adquisición y el estudio de ese repertorio caótico, pero no desconozco su variedad y el creciente ámbito de sus temas" (Evaristo Carriego; OC 1974: 163). Viñas cita o comprende mal el dictum de Borges en su conferencia inaugural del 17 de agosto de 1993: “Alguien dijo que para entender el tango esencial (da vergüenza decirlo: Borges) hay que leer la colección completa de El alma que canta." 12. El Plan de las Operaciones que el gobierno provisional de las Provincias Unidas del Río de la Plata debe poner en práctica para consolidar la grande obra de nuestra libertad e independencia está compuesto por una introducción y nueve artículos, y lleva fecha del 30 de agosto de 1810. Ya había motivos lingüísticos para duda de que Moreno fuese el autor de esta obra. Entre tanto, Diego Javier Bauso demostró que el texto fue fraguado en base a una novela francesa por allegados a la corona portuguesa, con el objetivo de desprestigiar a la Primera Junta e incitar a combatirla (2015). Noemí Goldman (2016) refuerza esa visión en Mariano Moreno. De reformista a insurgente. 13. Tras "don", varias palabras tachadas e ilegibles en el manuscrito. 14. "esclavos puestos al ser" en el manuscrito.

15. El texto entre corchetes falta en la versión impresa; lo completo en base al manuscrito. La lectura del pasaje es, empero, insegura, ya que la letra es en parte muy enrevesada; dos términos me resultan del todo ilegibles. 
16. En el manuscrito "en la Amalia de Mármol”.

17. En el manuscrito "congelad violadora". La "violación” es uno de los motivos que impregnan la obra de Viñas. Véase un detallado estudio de ese leitmotif en Valverde (1989: 227-245).
18. Dirigida por José Ingenieros y Leopoldo Lugones (1996 [1897]). locura, hasta entrecerrarse, notoriamente, entre lo civilizado y la barbarie. Dualismos que, si por una vertiente se nexan con la práctica de lo ensayístico en conjuro de lo ficcional, por el otro lado le permiten a Sarmiento proponerse como paradigma en polémica con Facundo. Dicotomía crítica pensada que valida el urbanismo victoriano en detrimento de la "ruralización americana".

Por cierto que este pivote dramático (que impregna y define, en un nuevo corte sincrónico, a los miembros de la llamada Generación del 37) encuentra su culminación simbólica en relación a Buenos Aires en la Amalia (1851-1855). ${ }^{16}$ Brevemente: la novela de Mármol puede ser leída como un "drama óptico" desde la mirada violadora ${ }^{17}$ de Rosas que se contrapone a los ojos descifradores del antagonista Daniel. Ambos, en esta perspectiva, pueden ser analizados como proyecciones del narrador omnisciente y consabido del siglo XX; y como Rosas y Daniel operan en función de deducciones a lo Sherlock Holmes (frente a un par de doctores Watson encarnados recíprocamente, en el cónsul inglés y en el francés), se podría decir que a la típica novela burguesa del 1850 solo le queda como alternativa textual la novela policial. Así como a Daniel no le queda otra alternativa -en su juego de dos pañosque trocarse en un flâneur que, como conoce minuciosamente Buenos Aires, realiza las reuniones de su "sociedad secreta" destinada a la liberación de la ciudad, en contraposición a la sociedad oficial, en un lugar tan clandestino como es el quilombo de la calle Cochabamba.

Se podría abundar. Pero para tratar de proseguir con el seguimiento de mi hipótesis: en Cambaceres, paradigma simbolizador del 1880 porteño, la pareja ciudad institucional / ciudad clandestina se verifica en lo que va desde el espacio del Club en dirección a la garçonière del protagonista de Sin rumbo. Así como la prolongación de ese interjuego fundamental se enhebra con la antinomia genealogía / prontuario que al dilatarse en la década Roca-Juárez Celman produce la dualidad Don / "alias", coreografía resuelta entre gentlemen y ladrones, algunas de cuyas declinaciones pasan por el lombrosiano Hombre de empresa o a través de Los ladrones de Buenos Aires y su manera de robar.

Incluso, y a los efectos de matizar este itinerario porteño, se podría insinuar, que en función de la dialéctica histórica mayor, el tramo simbólico que va de 1880 al 90, de La gran aldea hasta La Bolsa puede ser entendido como una introducción a la Ley de Residencia de 1902: el "otro" será el inmigrante europeo convocado desde la constitución de 1852 que, al no poder convertirse en exitoso ha caído en la cárcel o en la enfermedad, y [al] que apenas si le queda convertirse en merodeador de los "barrios altos" de Buenos Aires o, paródico, en un loco que se limita a imitar a los "picos de oro" de la gentry liberal.

Dando un paso adelante: el recinto urbano simbólico del escritor sobre el 1900 es la Torre de marfil: si bien adquiere diversas inflexiones al jugar en torno a una "altura lujosa" enfrentada a un contexto agresivo, la ecuación que predomina es la de Lugones con Las montañas de oro (1897): la altura adquiere aquí una dimensión geográfica, a la vez que lo ebúrneo decorativamente se endurece en oro. Por un lado. Porque por el otro flanco condensa las señales que Lugones pone en juego en esa "Exageración Jacobina" que sirve de título a su revista de esos años llamada La Montaña. ${ }^{18}$

Ahora bien, el doble diapasón que surge de ese lugar poético alude, al mismo tiempo, a un auditorio concreto de la ciudad en simetría respecto de otro auditorio ideal: se trata, hacia arriba, de los dioses con quienes Lugones se tutea en una especie de plegaria; hacia abajo, el mensaje lugoniano apela a "las masas", y son las órdenes que se desploman desde la cima de las montañas que van dibujando el típico espacio urbano del arrabal. 
Podría inferirse quizás, el circuito que ese ademán autoritario va recorriendo a lo largo de ese paradigma del 1900 porteño que es Lugones desde Las montañas de oro hasta el Roca (1938). El "orografismo" va resultando constante, aunque en la inversión de 180 grados que marca la dirección de Lugones al desplazarse desde los arrabales simbólicos hacia los "auditorios privilegiados" del teatro Odeón y del teatro Coliseo.

1924, en este itinerario personal lugoniano, se me aparece como el eje: es el año de su Discurso del sable, ${ }^{19}$ que a partir de su coloración mussoliniana, resuena a precursor, como apertura del tono fascista en el cada vez más polarizado espacio urbano porteño. Entonación inquietante que se va organizando en esa serie enfática que se abre entre La patria fuerte y La grande Argentina, en 1930.

En medida relación con aquella fecha y esta entonación lugoniana, y a lo largo del momento yrigoyenista que se extiende del 1916 al 1930, se ponen en la superficie los dos emblemas literarios que inauguran la simbólica moderna de Buenos Aires: Borges y Arlt (como emergentes, también del vanguardismo argentino de los años 20). Borges enunciando una voz que si se recorta sobre la de Lugones, se va definiendo en virtud de la incidencia de Macedonio Fernández: la frontalidad lugoniana entra en crisis con Borges, y el énfasis orográfico se atenúa en lo confidencial; la "corporalidad viril" de Lugones se troca en una literatura analgésica que va implicando tanto la elusión de los textos como el escamoteo corporal.

Es en esta peculiar sustracción donde se puede escribir el talante urbano predominante en el primer Borges, en quien la andadura se sobreimprime con la escritura: Fervor de Buenos Aires (1923) resulta así en su dimensión revés / derecho, el conjuro de lo urbano central y canónico en beneficio de un arrabal cada vez más matizado en su iluminación y menos inquietante en sus escenografías y en sus habitantes. Por más de una intuición, ${ }^{20}$ el predominio de lo tangencial de Carriego irá predominando aceleradamente al respecto de la centralidad lugoniana.

En lo que hace a Roberto Arlt en esta misma coyuntura, corresponde insinuar, por lo menos, la polémica [implícita en 1926, con el último representante de la literatura] ruralista: ${ }^{21}$ El juguete rabioso de Arlt, tanto por su paideia urbana como por su lenguaje, valores y escenarios, se sitúa en el extremo opuesto del aprendizaje campesino del Segundo Sombra. Incluso, si por su versión del trabajo, el Juguete se antagoniza con la mitología del trabajo materno instaurada por Sarmiento en Recuerdos, por su relación con el dinero polemiza con Lugones, por sus comentarios al sexo se aproxima al otro representante principal en ese momento (1926) que es Versos de una... de César Tiempo, para quien la puta de origen inmigrante es la antítesis urbana del protagonista de Güiraldes.

Para ir entrecerrando esta hipótesis de itinerario simbólico urbano, habría quizás que aludir al interjuego entre el "infierno" y el "cielo" en Rayuela de Cortázar de 1963: al fin de cuentas, su doble dimensión alude, mediatamente, al "despegue" desde la materialidad porteña en dirección a la espiritualización parisina. Ese viaje (además de culminar el itinerario tradicional desde el Buenos Aires de 1800 hacia "el centro del mundo"), pone nuevamente en escena el vaivén tan reiterado entre la cultura decorativa de fachada y la "miserable dimensión del contrafrente".

Rodolfo Walsh, en este orden de cosas, aparecerá como el emergente principal del corte sincrónico que se da en Buenos Aires desde el 1976 hasta el 1983: incluso su desplazamiento desde "el juego policial" (más o menos vinculado a Borges), en dirección a un documentalismo despojado de adjetivos, vendría a corroborar, desde una inflexión inédita en la que la heterodoxia llevada hasta el límite pone en escena la inexorable sanción, la polarización primordial de Buenos Aires. ${ }^{22}$
19. DV alude al discurso leído por Lugones en Ayacucho, donde habla de la "hora de la espada". El discurso, leído en Perú ante el dictador Leguía, alcanzó rápida difusión en Hispanoamérica. En cuanto a la Argentina, el discurso de Lugones fue una especie de prólogo a la década infame inaugurada con el golpe de Uriburu en septiembre de 1930.

20. En vez de "intuición", el manuscrito trae "razón", que conviene más al sentido de la frase.

21. El texto entre corchetes proviene del manuscrito; falta en la versión impresa. El pasaje alude a Güiraldes. 
23. "Zaunkönig" es el nombre de un pájaro pequeño y movedizo; en el libro, es un alter ego del autor.
24. Alusión al libro de Juan José Sebreli (1964): Buenos Aires: vida cotidiana y alienación. Fue reeditado por Sudamericana en 2003 , con un nuevo prólogo y aumentado con el texto "Buenos Aires, ciudad en crisis".
Así como, por ahora, ya en 1990 se enfrentan, polémicamente, el shopping Bullrich y el albergue Warnes; dos dimensiones: shopping y albergue que reactualizan una vez más la dialéctica dramática que, me parece, va sintetizando al máximo los emblemas de Buenos Aires.

David Viñas,

por borrador

19-VI-90

En el libro de Daus arriba citado se reproduce a continuación el texto de Reichardt ("Literatura de Buenos Aires"). Al final del volumen se recogen fragmentos de un diálogo final, pero no hay allí nada de Viñas; sí, una breve intervención de Reichardt.

Este informa acerca de ese congreso en Berlín, de una estadía de Viñas y Dalmiro Sáenz en su casa y sobre un seminario dado conjuntamente en Hamburg, en su diario novelado Zaunkönig (2015a: 451-454). ${ }^{23}$

Traduzco del alemán, a veces solo parafraseando, párrafos del apartado VIII del libro 6 de ese Diario, que lleva por título: "Comunidad de hombres. Final de la República Democrática Alemana. Coloquio sobre grandes ciudades. Dalmiro Sáenz y David Viñas en Bockenkistedt" (Bockenkistedt es un trasunto ficticio de Rhadereistedt, pequeña localidad situada entre Hamburg y Bremen, donde vivía Reichardt por esas fechas):

A mediados de junio de 1990 debía tener lugar en Berlín un Coloquio organizado por Ronald Daus acerca de las grandes ciudades extraeuropeas. A Zaunkönig le tocó en suerte el capítulo sobre Buenos Aires, y él pudo invitar hasta a tres autores a conferenciar sobre el tema. Ninguno parecía mejor calificado para ello que David Viñas; Zaunkönig pensó además en Juan José Sebreli, de quien apreciaba mucho el ingenioso ensayo sobre Buenos Aires, aunque este fue a menudo denostado. ${ }^{24}$ Como tercer participante deseó tener a Dalmiro Sáenz, ya que este había investigado los mundos desconocidos de los mataderos y de los sistemas de canalización subterránea, con sus buscadores de oro. David aceptó; Sebreli rechazó la invitación debido a una enfermedad; Dalmiro solo respondió a su carta certificada con una inusual, que apenas contenía un "Sí" manuscrito.

Después de narrar un paseo involuntario por Berlín oriental, prosigue Reichardt:

Zaunkönig se encontró en el hotel Domus con David y Dalmiro, a quien no solo le debía el placer de la carta más breve recibida en su vida, sino también la lectura de relatos y novelas que permitían reconocer a un autor que seguía un sendero muy original, independiente tanto de la intelectualidad existencialista de David como de lo vanguardístico de los herederos de Borges y Cortázar. El primer litro de vino corrió ya durante los saludos; a él seguirían muchos otros, de vinto tinto y blanco, en los 11 días que pasaron juntos.

(Sorprende este dato, ya que según opiniones recabadas en Buenos Aires en marzo de 2018 de personas que trataron a Viñas, este era más bien ascético en cuanto al alcohol.)

Reichardt relata que la sección "Buenos Aires" fue la menos fructífera, si bien suscitó asombro o alguna diversión al público. Cuando le tocó hablar, Dalmiro Sáenz había 
desaparecido, hasta que Christian Sönnichsen (alumno y colaborador de Reichardt, hoy traductor), lo encontró y lo convenció de que dijera cualquier cosa que tuviera que ver con una gran ciudad. Sáenz no había preparado ningún manuscrito, de modo que su ponencia fue improvisada y no muy coherente, para disgusto del público y de Viñas. Todo ello concurre a explicar por qué no hay texto alguno de Sáenz en el libro de Daus...

El regreso a Buenos Aires estaba planeado para una semana después del Coloquio, de modo que los visitantes argentinos pasaron a la casa de Reichardt, ubicada muy fuera de la ciudad. Pero esa es ya otra historia, menos académica.

(Hamburg, 1-IV-2018) 


\section{Bibliografía}

»Bauso, D. J. (2015). Un plagio bicentenario. El Plan de operaciones atribuido a Mariano Moreno. Mito y realidad. Buenos Aires.

»Canal Feijóo, B. (1979). Los fundadores. Buenos Aires: CEdAL.

»Daus, R. (ed.) (1992). Großstadtliteratur. Ein Internationales Colloquium über Lateinamerikanische, Afrikanische und Asiatische Metropolen in Berlin, 14.-16. Junio 1990. La literatura de las grandes ciudades / La littérature des grandes villes. Frankfurt am Main: Vervuert, pp. 77-88.

» El Jaber, L., Schmidel, U. (2016 [1938; 1950]). Derrotero y viaje a España y las Indias. Traducción: Edmundo Wernicke. Paraná: Editorial de la Universidad Nacional de Entre Ríos.

» García, C. (2017/03). “David Viñas y el Che: sobre una obra de teatro desconocida": www.academia.edu, subido el 22-III-2017. [Reseña de Viñas 2016]

" García, C. (2017/o8). “Historia de una ausencia: David Viñas, Rodolfo Walsh y Les Temps Modernes”: Revista electrónica Transas, Buenos Aires, 4-VIII-2017, rúbrica "Archivo"; Disponible en <http://www.revistatransas.com/2017/08/o4/ historia-de-una-ausencia-rodolfo-walsh-david-vinas-y-les-temps-modernes/>.

» García, C. (2017/12). “David Viñas y Tina Modotti, desnuda e invicta”: Boca de Sapo 25, Buenos Aires, diciembre de 2017, pp. 25-35; texto subido el 31-XII-2017: $<$ www.bocadesapo.com.ar/pdf/04\%20-\%20CarlosGarcia.pdf>.

» García, C., Reichardt, D. (2004). Las vanguardias literarias en Argentina, Uruguay y Paraguay. Bibliografía y antología crítica. Madrid / Frankfurt am Main: Iberoamericana / Vervuert.

" Goldman, N. (2016). Mariano Moreno. De reformista a insurgente. Buenos Aires: Edhasa.

»La Montaña. Periódico socialista revolucionario (1996 [1897]). Dirigido por José Ingenieros y Leopoldo Lugones. Bernal: Universidad Nacional de Quilmes.

"Lomba, A. (1998). El Alma que Canta. Buenos Aires. Academia Porteña del Lunfardo / Junta de Estudios Históricos del Barrio de Boedo.

"Monteleone, J. (2013). “La vaca o la res argentina”, en Comidas bastardas: Gastronomía, tradición e identidad en América Latina. Edición e introducción de Ángeles Mateo del Pino y Nieves Pascual Soler. Santiago de Chile: Cuarto propio.

» Obermeier, F., Schmidel, U. (2008). Reise in die La Plata-Gegend (1534-1554). Kritische Ausgabe. Kiel: Westensee Verlag (contiene también la traducción de Wernicke, en la versión de 1948, y numerosos apéndices).

» Reichardt, D. (2015a). Zaunkönig. Biographischer Roman. Heeslingen: Ei*Del*Hus.

"Reichardt, D. (2015b). Zaunkönigs Argentinien, 1973-87. Heeslingen: Ei*Del*Hus [Extracto de 2015a; no contiene todos los pasajes relacionados con Argentina].

"Tieffemberg, S. (ed.) (2012). Argentina. Historia del Descubrimiento y Conquista del Río de la Plata de Ruy Díaz de Guzmán. Edición crítica, prólogo y notas de ST. Buenos Aires: Editorial de la Facultad de Filosofía y Letras / Universidad de Buenos Aires.

"Tieffemberg, S. (ed.) (2012). El Romance de Luis de Miranda. Buenos Aires: Editorial de la Facultad de Filosofía y Letras / Universidad de Buenos Aires. 
» Tieffemberg, S. (ed.) (2014). Romance. Madrid: Vervuert (Biblioteca indiana, 38).

»Valverde, E. (1989). David Viñas: en busca de una síntesis de la historia argentina. Buenos Aires: Plus Ultra.

»Viñas, D. (2016). Del Che en la frontera / Che im Grenzland. Edición bilingüe. Zweisprachige Ausgabe. Editado y traducido por DR. Heeslingen: Ei*Del*Hus. [Cf. C. García 2017/03]. 
